\title{
Optical Nonlinearities in PbSe Nanocrystals
}

\author{
G. Aleksejenko, V. PaČebutas* and A. Krotkus
}

Semiconductor Physics Institute, A. Goštauto 11, 01108 Vilnius, Lithuania

The linear and nonlinear optical response of colloidal PbSe nanocrystals was investigated. Optical nonlinearity and its recovery dynamics in this system was measured and compared with other available experimental data. The saturation irradiance value for the bleaching effect and absorption cross-section were determined.

PACS numbers: 78.66.Jg, 42.70.Mp, 42.65.Re

\section{Introduction}

Nanometer-sized semiconductor nanocrystals (NC) exhibiting a large and fast recovering optical nonlinearity have been studied extensively as candidate materials for photonics applications and optical signal processing [1, 2]. Compared with well known $\mathrm{CdS}$ or CdSe semiconductor quantum dots, $\mathrm{NC}$ of $\mathrm{PbS}$ and $\mathrm{PbSe}$ are more attractive because of their larger exciton Bohr radius (46 nm for PbSe and $18 \mathrm{~nm}$ for $\mathrm{PbS}$ ). Therefore in PbSe or PbS nanocrystals one can expect much larger quantum confinement effect than in most other semiconductors.

There are several techniques proposed to fabricate NC of IV-VI semiconductors: process of the phosphate glass synthesis [3], colloidal chemistry [4]. Bigger attention was paid to investigation of nonlinear properties of $\mathrm{PbS} \mathrm{NC}$ obtained by the two of the mentioned above techniques. Much less attention was paid to PbSe. One could mention investigations of nonlinear optical response from this material in a phosphate glass [5].

In this work we give experimental investigations of nonlinear optical characteristics of colloidal PbSe NC immersed in hexane measured by using picosecond laser pulses and $z$-scan and pump-and-probe techniques.

\section{Experimental}

The samples were prepared filling a $529 \mu \mathrm{m}$ thick container with EviDots PbSe nanocrystal solution in hexane. Several different densities of PbSe nanocrys-

*corresponding author; e-mail: vaidas@opel2.pfi.lt 
tals in hexane were investigated. The first-exciton absorption peak was between 1000 and $1100 \mathrm{~nm}$.

The main results were obtained using experimental setup based on mode-locked Nd:glass laser (pulses duration $2.5 \mathrm{ps}$, pulse repetition rate $5 \mathrm{~Hz}$, wavelength $1054 \mathrm{~nm}$, maximum energy $22 \mu \mathrm{J})$. Nonlinear optical response of PbSe nanocrystals was measured by employing optical $z$-scan [6] and standard pump-and-probe techniques. All measurements presented in this paper were performed at room temperature.

\section{Results and discussion}

Measured linear absorption spectrum of the sample with PbSe concentration of $12.5 \mathrm{mg} / \mathrm{ml}$ is shown in Fig. 1. It has a peak typical of nanocrystals, which is due to first exciton resonance. The maximum value in our situation is at $\lambda=1.025 \mu \mathrm{m}$, however it depends on nanocrystals size and material constants. Using four-band envelope function formalism [7] it is possible to calculate diameter of the quantum dots. This theory gives us value of $5 \mathrm{~nm}$, but Lipovskii et al. [8] shows that this theory disagrees with experiment, when the size is smaller that $7 \mathrm{~nm}$. According to Lipovskii et al. the size of our nanocrystals is about $2 \mathrm{~nm}$ in diameter.

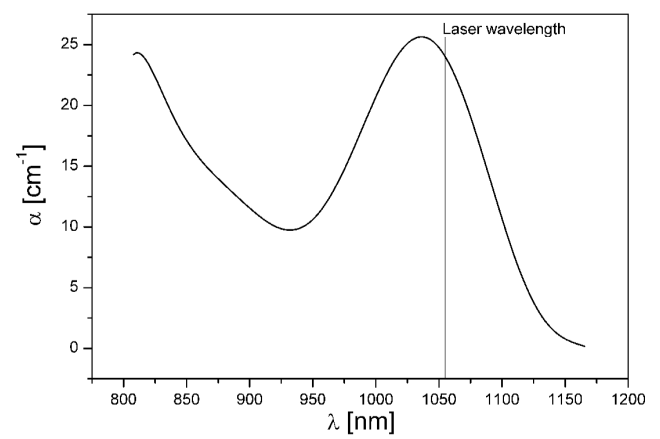

Fig. 1. Measured linear absorption spectrum of a sample with NC concentration $12.5 \mathrm{mg} / \mathrm{ml}$.

Next, we have investigated the nonlinear absorption at laser wavelength close to this resonance using open aperture $z$-scan. The magnitudes of light induced absorption bleaching as large as $60 \%$ were observed experimentally. The measured traces for two PbSe solutions in hexane with different density were used for plotting of the optical transmittance dependence on the irradiation. The results are presented in Fig. 2.

Using a simple two-level model [9], a transmittance versus irradiation dependence can be determined by using an intensity dependent absorption coefficient

$$
\alpha(I)=\frac{\alpha_{0}}{1+\left(I / I_{\mathrm{s}}\right)}+\alpha_{\mathrm{ns}} .
$$




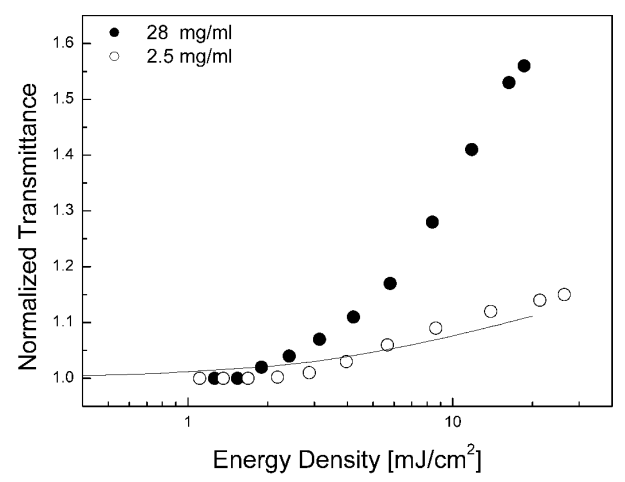

Fig. 2. Optical transmittance dependence on the irradiation for two different PbSe nanocrystal concentrations. The solid line — calculations results for $2.5 \mathrm{mg} / \mathrm{ml}$ case.

Here $\alpha_{\mathrm{ns}}$ is the value of the non-saturable part of the absorption coefficient, $\alpha_{0}$ is the saturable part of this coefficient at low optical intensities, and $I_{\mathrm{s}}$ is the saturation intensity. The latter parameter can be estimated from the threshold condition for the light-induced bleaching - average number of the photons absorbed in a single nanocrystal at the threshold light intensity should be close to one

$$
I_{\mathrm{s}} \approx \frac{h \nu}{\sigma_{0}}
$$

( $h \nu$ is the photon energy quantum, $\sigma_{0}$ is the optical absorption cross-section of the nanocrystals). Using value of $\mathrm{NC}$ diameter from above, we estimated density of nanocrystals $\left(8 \times 10^{16} \mathrm{~cm}^{-3}\right)$ and optical cross-section $\sigma_{0}=3 \times 10^{-17} \mathrm{~cm}^{2}$. This value is much smaller than the geometrical cross-section of the nanocrystals $\left(3 \times 10^{-14} \mathrm{~cm}^{2}\right)$. After inserting the value of $\sigma_{0}$ into $(2)$ we find $I_{\mathrm{s}}=6 \mathrm{~mJ} / \mathrm{cm}^{2}$.

Now we can calculate the dependence of the transmittance on the irradiance using Eq. (1). The result is shown in Fig. 2 by solid line. Good coincidence of experimental dependence with calculation can be seen for the solution with the PbSe nanocrystal density of $2.5 \mathrm{mg} / \mathrm{ml}$. When the concentration of $\mathrm{NC}$ increases this congruence disappears. Even so, simple two-level model gives a quantitative description of the experimentally observed nonlinear optical response of $\mathrm{PbSe}$ nanocrystals and thus can be used by designing photonic components using those nanocrystals.

The results of pump and probe transmittivity experiment are presented in Fig. 3. The shape of the temporal dependences measured on two solutions with different nanocrystal densities is similar. Fast decay characterized by a time constant of approximately $11 \mathrm{ps}$ is followed by much slower (of the order of $1 \mathrm{~ns}$ ) relaxation of the pump-induced bleaching. These values are in a good agreement with the results obtained in [5], despite the fact that the laser wavelength and matrix surrounding PbSe nanocrystals used in this work were different from the ones used in this investigation. One could assume, therefore, that at least the 


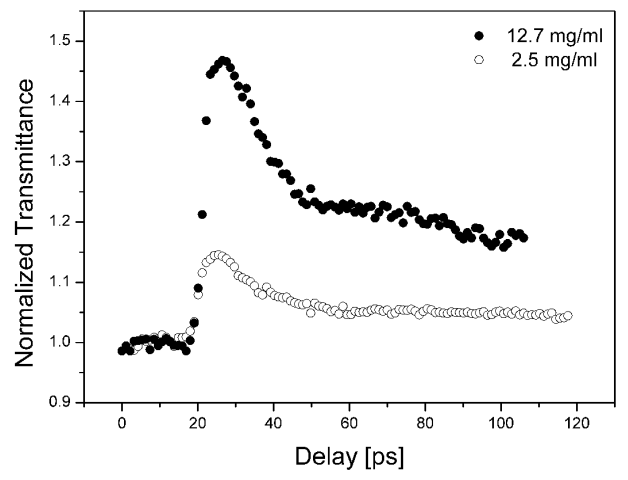

Fig. 3. Pump-and-probe transmittance traces for different PbSe nanocrystal concentrations. Pump energy $-7.3 \mu \mathrm{J}$.

shorter time constant is caused by intrinsic recombination processes, like radiative recombination, taking place in the nanocrystals.

\section{Conclusions}

The nonlinear optical response of PbSe nanocrystals embedded in hexane matrix using stationary $z$-scan and pump-and-probe transmittivity techniques were investigated. The value of the order of $6 \mathrm{~mJ} / \mathrm{cm}^{2}$ saturation irradiance for the bleaching effect was estimated. It was found that temporal decay of the optically induced bleaching was two-exponential, with the shorter time constant equal to $11 \mathrm{ps}$ independent of the excitation intensity and of the nanocrystals density in the solution.

\section{References}

[1] K. Wundke, S. Potting, J. Auxier, A. Schulzgen, N. Peyghambarian, N.F. Borrelli, Appl. Phys. Lett. 76, 10 (2000).

[2] G. Tamulaitis, V. Gulbinas, G. Kodis, A. Dementjev, L. Valkunas, I. Motchalov, H. Raaben, J. Appl. Phys. 88, 178 (2000).

[3] A.M. Malyarevich, I.A. Denisov, V.G. Savitsky, K.V. Yumashev, A.A. Lipovskii, Appl. Opt. 39, 4345 (2000).

[4] A. Hache, S.-E. LeBlanc, M. LoCascio, A. Martucci, Physica E 17, 104 (2003).

[5] T. Okuno, Y. Masumoto, M. Ikezawa, T. Ogawa, Appl. Phys. Lett. 77, 504 (2000).

[6] A.A. Said, M. Sheik-Bahae, D.J. Hagan, T.H. Wei, J. Wang, J. Young, E.W. Van Stryland, J. Opt. Soc. Am. B 9, 405 (1992).

[7] I. Kang, F.W. Wise, J. Opt. Soc. Am. B 14, 1632 (1997).

[8] A. Lipovskii, E. Kolobkova, V. Petrikov, I. Kang, A. Olkhovets, T. Krauss, M. Thomas, J. Silcox, F. Wise, Appl. Phys. Lett. 71, 2406 (1997).

[9] P.T. Guerreiro, S. Ten, N.F. Borrelli, J. Butty, G.E. Jabbour, N. Peyghambarian, Appl. Phys. Lett. 71, 1595 (1997). 